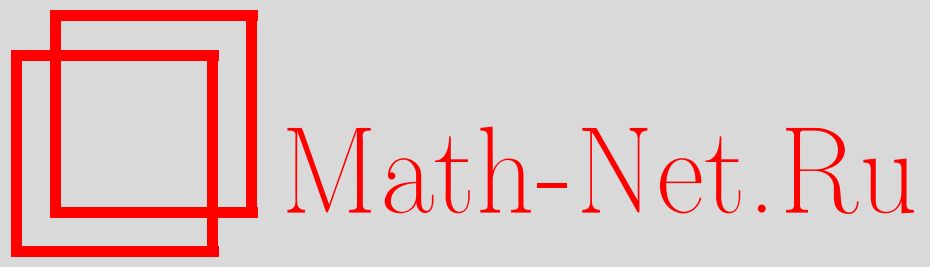

П. Панов, Квадратичные отображения в картинках (окончание), Квант, 2018, номер 8, 12-16

DOI: https://doi.org/10.4213/kvant20180802

Использование Общероссийского математического портала Math-Net.Ru подразумевает, что вы прочитали и согласны с пользовательским соглашением

http://www . mathnet.ru/rus/agreement

Параметры загрузки:

IP : 52.6 .47 .48

26 апреля 2023 г., $17: 04: 27$

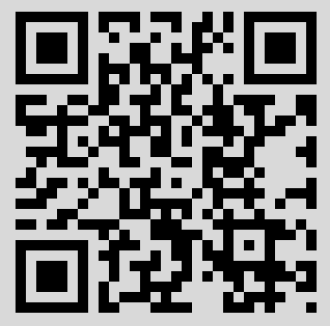




\section{Квадратичные отображения в картинках}

\section{П.ПАНОВ}

\section{Бифуркационная диаграмма}

Не так уж просто разобраться в многообразии типов долгосрочного поведения, которое демонстрирует квадратичное семейство $f_{\lambda}(x)=\lambda x(1-x)$. Но существует удобный способ одновременного представления динамики семейства $f_{\lambda}$ на одной картинке - это бифуркащионная диаграмма.

Возьмем диапазон изменения $\lambda$, в нашем случае отрезок $[0 ; 4]$, и равномерно расположим на нем большое количество точек $\lambda_{i}=4 i / I, i=0, \ldots, I$. Для каждого $i$ случайным образом выберем начальную точку $x_{0} \in[0 ; 1]$ и произведем большое количество $N$ итераций

$$
x_{n+1}=\lambda_{i} x_{n}\left(1-x_{n}\right) \text {. }
$$

Затем на координатной плоскости отметим точки $\left(\lambda_{i} ; x_{n}\right)$, где $n$ меняется от 100 до $N$. Все они будут лежать на одном вертикальном отрезке с абсциссой $\lambda_{i}$. Обратите внимание, поскольку нас интересует установившееся (долгосрочное) поведение точек $x_{n}$, первые 100 из них были отброшены. Проделаем это для каждого $i$.

Полученная таким образом бифуркационная диаграмма изображена на рисунке 3 $(0 \leq \lambda \leq 4)$. Тут можно четко различить те типы поведения, которые мы зафиксировали раньше. Например, то, что при $0 \leq \lambda \leq 1$ точки $x_{n}$ стремятся к 0 , а при $1<\lambda \leq 3-$ к некоторому ненулевому значению, а именно к корню $x_{\lambda}=(1-\lambda) / \lambda$. Видно появление цикла длины 2 при переходе $\lambda$ через значение 3 , а также виден цикл длины 4 при $\lambda=3,5$. Для тех $\lambda_{i}$, для которых заполнены целые отрезки, харак-

DOI: https://doi.org/10.4213/kvant20180802

Окончание. Начало - в предыдущем номере журнала.

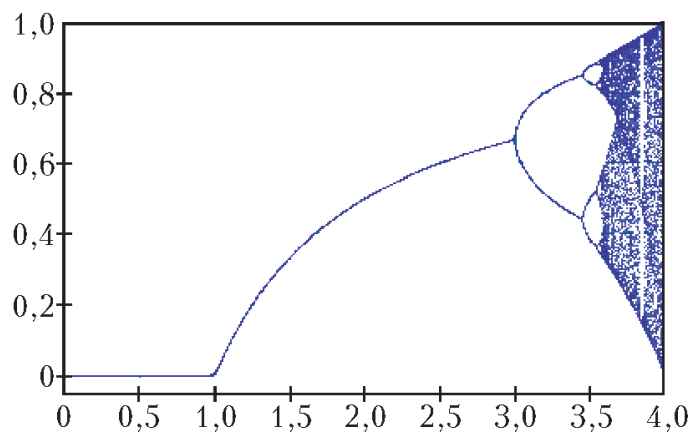

Pис. 3

терна хаотическая динамика. Где-то в районе $\lambda=3,8$ наблюдается цикл длины 3 . Разглядывая бифуркационную диаграмму при большем увеличении, можно обнаружить другие подробности.

Мы не можем продвинуться вдоль бифуркационной диаграммы в сторону значений $\lambda>4$, так как там при итерациях точки начинают уходить на бесконечность, но продвижение в сторону отрицательных значений возможно (рис. $4 ;-2 \leq \lambda \leq 4$ ).

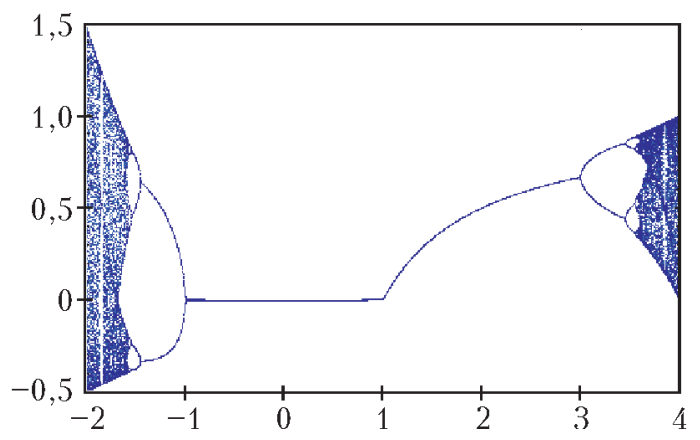

Pnc. 4

Дальше мы попробуем расширить диапазон изменения параметра $\lambda$ «вправо», научившись работать с точками, уходящими при итерациях на бесконечность, и 
построим новые бифуркационные диаграммы.

\section{Квадратичные отображения плоскости и отображения окружности, семейства и диаграммы}

Квадратичным отображением плоскости назовем преобразование, при котором точка с координатами $(x ; y)$ переходит в точку $A(x ; y)$ с координатами

$$
\begin{aligned}
& A(x ; y)= \\
& =\left(a_{1} x^{2}+a_{2} x y+a_{3} y^{2} ; a_{4} x^{2}+a_{5} x y+a_{6} y^{2}\right),
\end{aligned}
$$

где $a_{i}$ - некоторые фиксированные числа. Дальше при задании квадратичного отображения $A(x ; y)$ мы иногда будем пользоваться короткой записью $A\left(a_{1} ; a_{2} ; a_{3} ; a_{4}\right.$; $\left.a_{5} ; a_{6}\right)$, оставляя лишь его коэффициенты $a_{i}$.

С каждым преобразованием плоскости $A: \mathbb{R}^{2} \rightarrow \mathbb{R}^{2}$ можно связать преобразование окружности, которое на координатном языке выглядит как

$$
(x ; y) \mapsto \frac{A(x ; y)}{|A(x ; y)|} .
$$

Здесь $(x ; y)$ - точка на единичной окружности (т.е. $\left.x^{2}+y^{2}=1\right)$. Результат применения к ней преобразования, $A(x ; y)$, делится на расстояние до начала координат (нормируется), и новая точка возвращается на единичную окружность.

Итак, преобразование плоскости $A(x ; y)$ порождает преобразование окружности, и мы можем наблюдать его динамику, как это делали раньше для отрезка. Но давайте сразу перейдем к семействам и к их бифуркационным диаграммам.

Пусть имеются два квадратичных отображения $A$ и $B$. Они порождают естественное семейство квадратичных отображений

$$
C_{\lambda}=\lambda A+B, \quad-\infty<\lambda<\infty .
$$

Чтобы избавиться от бесконечного диапазона изменения $\lambda$, сделаем еще замену $\lambda=\sin \theta / \cos \theta$ и распределим $\sin \theta$ и $\cos \theta$ между $A$ и $B$ :

$$
C_{\theta}=A \sin \theta+B \cos \theta, \quad-\pi \leq \theta \leq \pi .
$$

Вот для таких семейств мы и построим сейчас несколько бифуркационных диаграмм. Первая из них, для семейства, порожденного $A=(-0,43 ; 0,06 ; 0,67 ;-0,53$; $-0,54 ;-0,47)$ и $B=(-0,57 ; 1,32,-0,29 ; 0$; $-0,94 ;-034)$, изображена на рисунке 5.

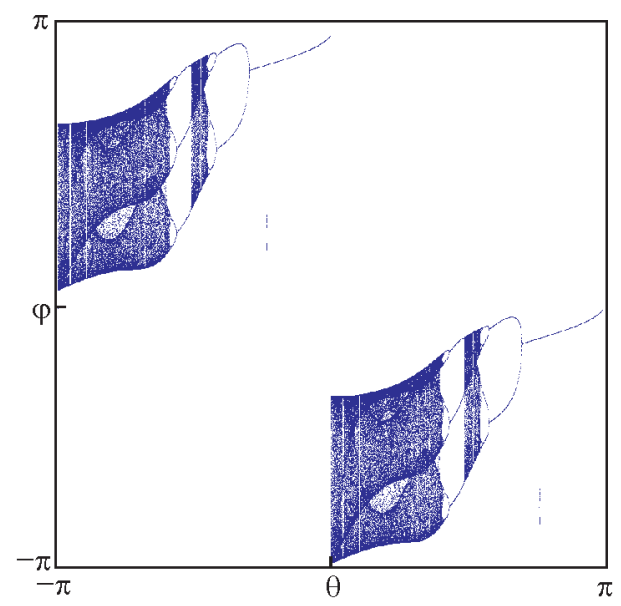

Puc. 5

На этом рисунке, как и на следующих, вдоль горизонтали откладывается параметр $-\pi \leq \theta \leq \pi$, а вдоль вертикали угловые координаты $-\pi \leq \varphi \leq \pi$ точек окружности, с которых стартует итерация (угловая координата $\varphi$ - это угол между осью $x$ и направлением на точку окружности).

На рисунке 5 опять наблюдаются бифуркации удвоения периода, видно периодическое и хаотическое поведение.

На окружности углам, отличающимся друг от друга на $2 \pi$, отвечает одна и та же точка, поэтому мы можем отождествить вертикальные стороны граничного квадрата, соответствующие углам $\theta= \pm \pi$. Точно так же можно отождествить и горизонтальные стороны, соответствующие $\varphi= \pm \pi$. Но на рисунке 5 имеется и еще одна дополнительная симметрия, которая заключается в том, что бифуркационная диаграмма переходит сама в себя при сдвиге на векторы $(\pi / 2 ; \pm \pi / 2)$. Это следствие квадратичности отображений $A$ и $B$.

На рисунке $6(A=(-0,67 ; 0 ; 0,63 ; 0,07$; $0,14 ; 0,73)$ и $B=(0,58 ; 0,86 ;-0,01 ;-0,52$; $-0,08 ;-0,2))$ мы видим новые элементы - 


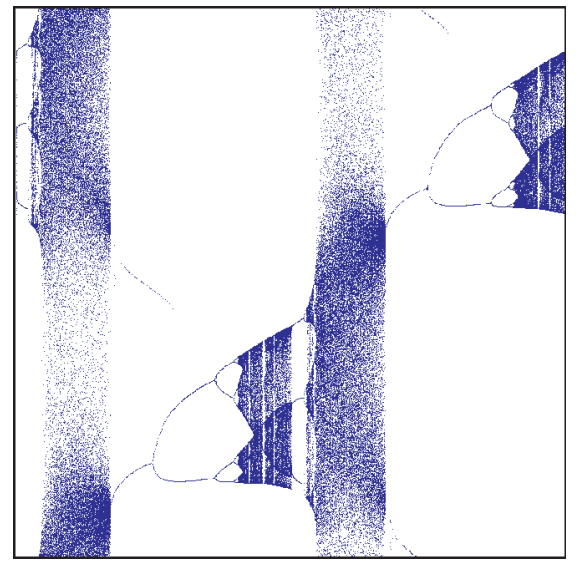

Pис. 6

вертикальные полосы. Они соответствуют тем значениям параметра $\theta$, для которых при отображении $C_{\theta}=A \sin \theta+B \cos \theta$ единичная окружность дважды наматывается сама на себя.

На рисунке 7 ( $A=(0,41 ;-1,12 ; 0,2 ; 1,06$; $0,94 ; 0,48)$ и $B=(0,86 ;-0,01 ; 1,07 ; 0 ; 0,22$; $0,14))$ - еще одна бифуркационная диа-

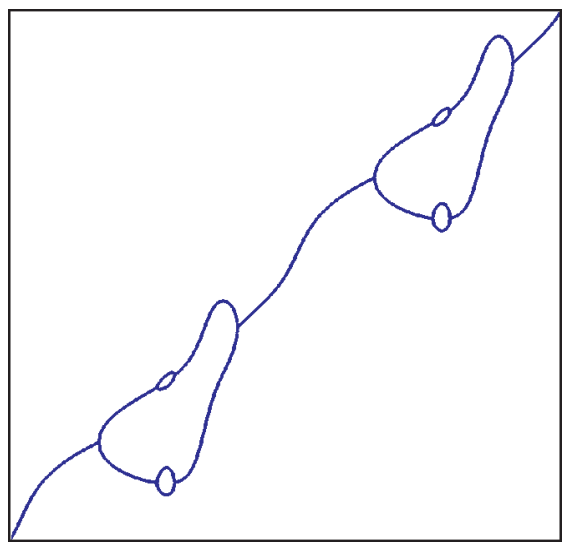

PUC. 7

грамма, на этот раз без какой бы то ни было хаотической компоненты. В наличии только неподвижные точки, циклы длины 2 и 4.

И наконец, возвращаемся к логистическому семейству. Оказывается, что оно напрямую связано с семейством, порожденным квадратичными отображениями $A(x ; y)=\left(0 ; x y-y^{2}\right)$ и $B(x ; y)=\left(x^{2} ; 0\right)$. Соответствующая диаграмма изображена на рисунке $8 \quad(A=(0 ; 0 ; 0 ; 0 ; 1 ;-1)$ и $B=(1 ; 0 ; 0 ; 0 ; 0 ; 0))$. Сравнение с рисунками 3 и 4 показывает, что в центре рисунка 8 фактически присутствует логистическая бифуркационная диаграмма.

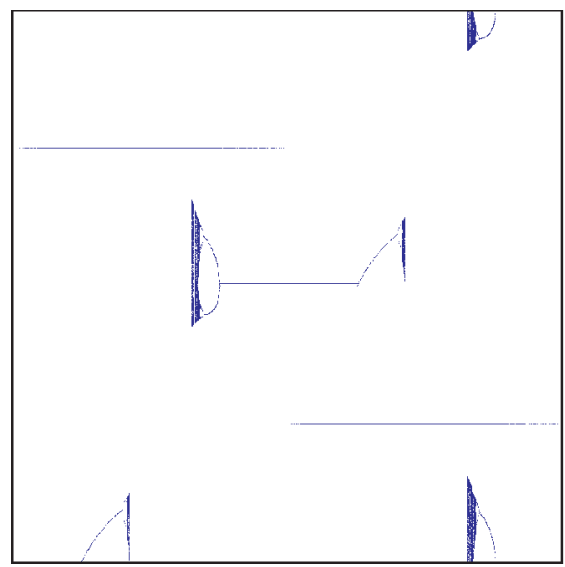

Pис. 8

Переход от координат $\theta, \varphi$ к координатам $x=\operatorname{tg} \theta, y=\operatorname{tg} \varphi$ дает точное соответствие между центральной частью рисунка 8 и рисунком 4 .

\section{Отображения сферы}

Тот самый фокус по превращению квадратичного преобразования плоскости в преобразование окружности мы повторим для пространства и сферы.

Квадратичным отображением трехмерного пространства назовем отображение $A: \mathbb{R}^{3} \rightarrow \mathbb{R}^{3}$ вида

$$
\begin{aligned}
& A(x ; y ; z)= \\
& =\left(a_{1} x^{2}+a_{2} x y+a_{3} x z+a_{4} y^{2}+a_{5} y z+a_{6} z^{2} ;\right. \\
& a_{7} x^{2}+a_{8} x y+a_{9} x z+a_{10} y^{2}+a_{11} y z+a_{12} z^{2} ; \\
& \left.a_{13} x^{2}+a_{14} x y+a_{15} x z+a_{16} y^{2}+a_{17} y z+a_{18} z^{2}\right) .
\end{aligned}
$$

Пусть точка $(x ; y ; z)$ лежит на единичной сфере с центром в начале координат, заданной уравнением $x^{2}+y^{2}+z^{2}=1$. Тогда точка $A(x ; y ; z) /|A(x ; y ; z)|$ тоже лежит этой сфере, и мы получаем отображение единичной сферы в себя:

$$
(x ; y ; z) \mapsto \frac{A(x ; y ; z)}{|A(x ; y ; z)|} .
$$


Опять можно взять точку на сфере и посмотреть, что с ней происходит при многократном применении этого отображения, т.е. проследить за динамикой точек $\left(x_{n} ; y_{n} ; z_{n}\right)$.

Ограничимся одним примером. Рассмотрим квадратичное преобразование $A$, показанное на рисунке 9. чительным стеклом, повторяют все изображение. ${ }^{4}$

В заключение скажем, что исследование поведения последовательности $x_{n}$ в методе простых итераций $\left(x_{n+1}=f\left(x_{n}\right)\right)$ служит не только для создания эстетически приятных рисунков. Даже если мы оказа-

$A=\left(\begin{array}{llllll}a_{1} & a_{2} & a_{3} & a_{4} & a_{5} & a_{6} \\ a_{7} & a_{8} & a_{9} & a_{10} & a_{11} & a_{12} \\ a_{13} & a_{14} & a_{15} & a_{16} & a_{17} & a_{18}\end{array}\right)=\left(\begin{array}{rrrrrr}-0,0879 & 0,9162 & 1,0522 & -0,1219 & 0,0408 & -1,0186 \\ 0,5917 & -0,0210 & -0,3582 & 0,4880 & -0,6976 & -0,4976 \\ -0,5585 & 0,4328 & 0,1542 & -1,0366 & 0,6024 & -0,1356\end{array}\right)$.

PUC. 9

Рисунок 10, напоминающий танцующую пару рыб, получен в результате 450000 итераций отображения единичной сферы,

\section{Pис. 10}

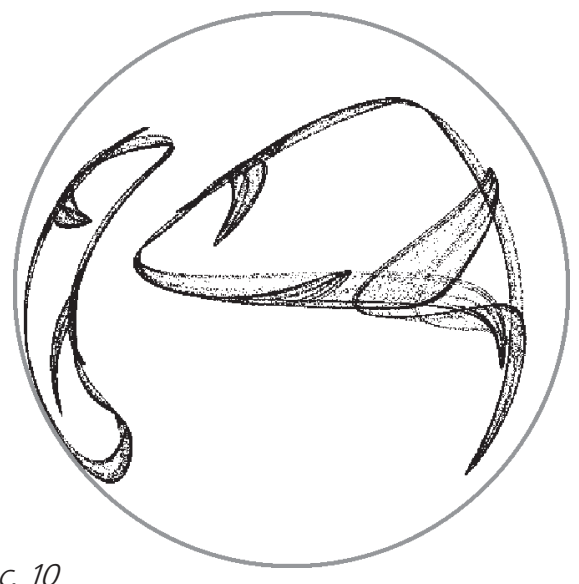

порожденного $A$. Каждая из последовательно полученных точек сферы $\left(x_{n} ; y_{n} ; z_{n}\right)$ была спроецирована на плоскость: $\left(x_{n} ; y_{n} ; z_{n}\right) \mapsto\left(x_{n} ; y_{n}\right)$. Все точки $\left(x_{n} ; y_{n}\right)$ лежат внутри единичного круга.

\section{Заключение}

Итак, на нескольких примерах мы увидели, что системы с очень простым описанием могут демонстрировать весьма нетривиальное поведение и «рисовать» сложные и красивые изображения со множеством деталей. Разумеется, в этой статье приведено лишь небольшое число таких картинок. Некоторое усложнение таких систем позволяет, например, рисовать фракталы - изображения, малые части которых, будучи рассмотрены под увели- лись в ситуации, когда этот метод не сходится к точному решению уравнения $f(x)=x$, бывает важно понять, что всетаки происходит.

Один из примеров такой задачи, приходящей из экономики, связан с паутинообразной моделью ченообразования. ${ }^{5}$ Вкратце, речь идет вот о чем. В капиталистическом обществе цены устанавливаются самими участниками рынка. Начнем с действующих в данное время цен $x_{0}$. Исходя из этих цен, производители и продавцы товаров стараются оптимизировать свою деятельность, после чего цены, выставляемые ими на товары, меняются: $x_{1}=f\left(x_{0}\right)$, и этот процесс продолжается дальше: $x_{2}=f\left(x_{1}\right)$, $x_{3}=f\left(x_{2}\right), \ldots{ }^{6} \mathrm{~B}$ «хорошем» случае итерационный процесс сойдется к уровню цен $x$, удовлетворяющему уравнению $x=f(x)$. Такие цены называются равновесными: даже если они кому-то покажутся несправедливыми, ни одному из участников рынка не выгодно их менять.

\footnotetext{
${ }^{4} \mathrm{O}$ том, как поведение динамических систем порождает фракталы, см. в книге: Х.О.Пайтген, П.Х.Рихтер. Красота фракталов. - М.: Мир, 1993.

5 Более подробно см., например: В.М.Гальперин, С.М.Игнатьев, В.И.Моргунов. Микроэкономика. - СПб.: Экономическая школа, 2004.

6 Для простоты модели мы предполагаем, что экономическая деятельность осуществляется дискретно, по шагам, как в некоторых компьютерных играх-стратегиях.
} 
В реальности, как мы видели в этой статье, так происходит не всегда, даже если наша экономическая модель (т.е. функция $f$ ) описана простой и понятной формулой. Может возникнуть и периодическое, и хаотическое поведение.

Более классические примеры связаны с динамическими системами в механике. Уравнения, описывающие поведение ме- ханических систем, довольно простые (грубо говоря, они получаются из законов Ньютона), однако поведение может оказаться очень сложным. Основы теории динамических систем заложил выдающийся французский математик А.Пуанкаре; впоследствии большой вклад в нее внесли А.Н.Колмогоров, В.И.Арнольд и другие.

\section{Н А М П И Ш У Т}

\section{Сечение с наименьшей площадью}

В последних номерах «Кванта» было опубликовано несколько статей под общим заглавием «Где ошибка?». Нам кажется очень важным и полезным тренироваться отличать правильные рассуждения от правдоподобных. Продолжая эту тему, наш постоянный автор и читатель С.Дворянинов предлагает протестировать на правильность решение следующей задачи.

Задача. Координать вершин треугольной пирамиды ТАВС таковы:

$$
T(2 ; 1 ; 0), A(0 ; 0 ; 1), B(1 ; 1 ; 0), C(0 ; 0 ; 2) \text {. }
$$

Пусть $S$ - это площадь сечения пирамидь плоскостью, проходящей через сторону основания АС и пересекающей боковое ребро ТВ. Найдите наименьшее значение величины $S$.

Решение. Рассмотрим сечение $A M C$ (см. рисунок). Пусть $M K$ - высота треугольника

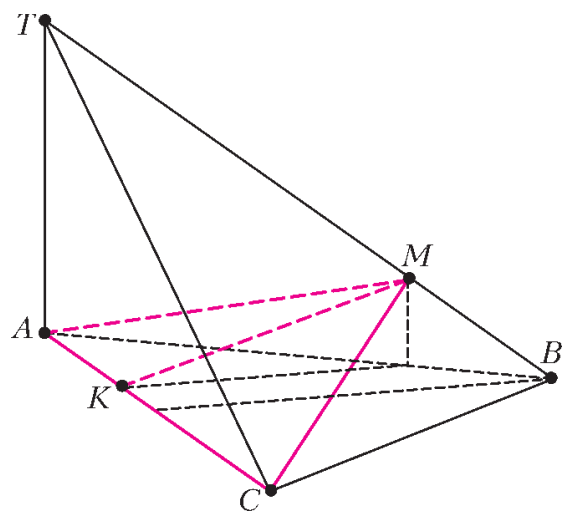

AMC. Площадь треугольника окажется наименьшей, когда будет наименьшей длина отрезка $M K$. В свою очередь, эта длина является наименьшей из всех возможных, если она равна расстоянию между скрещивающимися прямыми $A C$ и $T B$. Найдем это расстояние.

Прямая $A C$ задается уравнениями $x=0$, $y=0, z=t$, прямая $B T$ задается уравнениями $x=s, y=1, z=0$. Квадрат расстояния между точками этих двух прямых равен

$$
d^{2}=s^{2}+1^{2}+t^{2} \geq 1 .
$$

Равенство достигается при $s=0$ и $t=0$. Отсюда следует, что расстояние между прямыми $A C$ и $B T$ равно 1. Длина ребра $A C$ тоже равна 1. Следовательно, наименьшее значение $S$ равно $\frac{1}{2}$.

Все ли верно в этом решении?

В приведенном рассуждении имеется пробел, связанный с тем, что одно из оснований общего перпендикуляра к прямым $A C$ и $B T$ может находиться не на отрезке $B T$, а на его продолжении. Можно проверить, что при данных в условии задачи числовых значениях именно так и происходит. 